\title{
Tobacco control in the Eastern Mediterranean region: implementation progress and persisting challenges
}

\author{
Niveen M E Abu-Rmeileh (D) , 'Yousef Saleh Khader, ${ }^{2}$ Hanan Abdul Rahim, ${ }^{3}$ \\ Aya Mostafa (1) , ${ }^{4}$ Rima T Nakkash (1) , ${ }^{5}$ Randah R Hamadeh (D) , \\ Habiba Ben Romdhane, ${ }^{7}$ Ramzi George Salloum (D) ${ }^{5,8}$
}

\begin{abstract}
- Additional supplemental material is published online only. To view, please visit the journal online (http://dx.doi. org/10.1136/tobaccocontrol2021-056539).
\end{abstract}

${ }^{1}$ Institute of Community and Public Health, Birzeit University, Birzeit, Palestine, State of ${ }^{2}$ Epidemiology and Biostatistics, Jordan University of Science and Technology, Irbid, Jordan ${ }^{3}$ College of Health Sciences, QU Health, Qatar University, Doha, Ad Dawhah, Qatar ${ }^{4}$ Department of Community, Environmental, and Occupational Medicine, Faculty of Medicine, Eid Shams University, Cairo, Egypt ${ }^{5}$ Faculty of Health Sciences, American University of Beirut, Beirut, Lebanon

${ }^{6}$ College of Medicine and Medical Sciences (CMMS), Arabian Gulf University, Manama, Bahrain

${ }^{7}$ Faculty of Medicine, University of Tunis El Manar, Tunis, Tunisia ${ }^{8}$ Health Outcomes and Policy, University of Florida, Gainesville, Florida, USA

Correspondence to Dr Niveen M E Abu-Rmeileh, Institute of Community and Public Health, Birzeit University, 627 Birzeit, Palestine, State of; nrmeileh@birzeit.edu

Check for updates

(c) Author(s) (or their employer(s)) 2022. No commercial re-use. See rights and permissions. Published by BMJ.

To cite: Abu-Rmeileh NME, Khader YS, Abdul Rahim H, et al. Tob Control

2022:31:150-152
Tobacco use is the leading cause of preventable mortality worldwide, projected to claim 1 billion lives in the twenty-first century. ${ }^{1}$ Tobacco prevalence rates are expected to decrease across all the WHO regions by 2025 as a result of tobacco control efforts. However, the smallest decline is projected in the Eastern Mediterranean region (EMR)from $33.3 \%$ in 2020 to $31.0 \%$ in 2025 -making it almost certain that the region will not achieve WHO's 30\% relative reduction target by 2025.

The EMR includes 22 member states, with a total population of nearly 680 million that is diverse in terms of demographic, geographic, political and socioeconomic characteristics. The EMR has a history of conflict and protracted political and humanitarian crises. These unstable conditions, in addition to underdeveloped public health systems, ${ }^{2}$ have undermined tobacco control efforts and contributed to the persistence of weak tobacco regulatory environments across countries in the region. ${ }^{3}$ Moreover, about onethird of the region's population is between the ages of 15 and 29 years, ${ }^{4}$ representing a critical subgroup particularly susceptible to tobacco marketing.

According to WHO estimates in $2021,{ }^{1}$ the agestandardised current tobacco smoking prevalence among individuals aged 15 years or older in the region ranged from $8.1 \%$ in Oman to $35.0 \%$ in Jordan and Lebanon. Among men, the rates were highest in Jordan (57.1\%), Egypt (47.6\%), Tunisia (45.4\%) and Lebanon (41.63\%), and lowest in Oman (16.0\%). Prevalence of tobacco smoking is markedly lower among women, ranging from $0.3 \%$ in Oman to $28.2 \%$ in Lebanon and $12.9 \%$ in Jordan (online supplemental table). ${ }^{1}$ Waterpipe tobacco smoking has spread rapidly in the region over the past three decades, especially among young people in the region. ${ }^{5}$ Although waterpipe smoking prevalence is higher among men in most EMR countries, the gender gap is smaller than that for cigarette smoking. ${ }^{5}$ Various factors have contributed to the rise in waterpipe smoking rates, including the introduction of flavoured tobacco, the perception of reduced harm and addiction relative to cigarette smoking, and inadequate policies and regulations addressing waterpipe smoking. ${ }^{6}$

Among youth (13-15 years old), current tobacco smoking rates range from $3.7 \%$ in Oman to $28.1 \%$ in the occupied Palestinian territory. Cigarette smoking is the most common form of tobacco use, and it is highest in Palestine (17.5\%), followed by Bahrain (13.4\%), Kuwait (11.6\%) and Lebanon (11.2\%). The gender gap observed in adults is also seen in youth, but appears smaller for smokeless tobacco and e-cigarettes, although trends were difficult to discern with only 11 countries reporting recent data (within the past 5 years). Only four countries reported e-cigarette use from the Global Youth Tobacco Survey: Yemen (14.5\%), Qatar (11.3\%), Iraq (7.5\%), and Morocco (5.3\%).

Across the region, 19 countries have ratified the WHO Framework Convention on Tobacco Control (FCTC), and many have passed supporting legislation. $^{7}$ Overall, EMR countries have experienced partial success in FCTC implementation, but many challenges persist, especially in the enforcement of tobacco control measures and the adaptation of legislation and regulation to address novel tobacco products. We discuss here examples of implementation success and remaining challenges, organised by articles of the FCTC.

\section{ARTICLE 6. PRICE AND TAX MEASURES TO REDUCE THE DEMAND FOR TOBACCO}

Thirteen EMR countries have total taxes accounting for more than $50 \%$ of the retail price, second only to the European region where more than $88 \%$ of countries fall in this category. The six Gulf Cooperation Council (GCC) countries (ie, Bahrain, Kuwait, Oman, Qatar, Saudi Arabia and the United Arab Emirates) have implemented a unified tax increase plan which creates the framework for the implementation of value-added taxes across all member states. ${ }^{8}$ Egypt has also implemented steady annual tax increases since 2010 that have exceeded the WHO-recommended $75 \%$ of the retail price. ${ }^{1}$ A recent report examining performance of cigarette tax policies globally found that Bahrain, Saudi Arabia and the United Arab Emirates had the greatest improvements in their cigarette tax score, while the region as a whole had the second lowest score globally. ${ }^{9}$ While the report focused on cigarette tax policy, another challenge for countries in the region is that waterpipe tobacco products are not taxed at the same levels as cigarettes. ${ }^{10}$ Given the complexity of the tobacco product landscape in this region, further research is needed to assess which population subgroups are most affected by tax increases, especially considering vulnerable populations.

\section{ARTICLE 8. PROTECTION FROM EXPOSURE TO TOBACCO SMOKE}

The EMR ranks last among all WHO regions in implementing smoking bans in public places with 
only 15 countries having implemented partial ban policies. Whereas Jordan and Lebanon have implemented smoke free legislation in public places, many EMR countries still do not ban smoking in public places (eg, Bahrain, Oman, Somalia, Sudan and Tunisia). In Jordan, the ban was instituted in 2011, but faced implementation challenges due to resistance from several stakeholders, including the hospitality sector. ${ }^{11}$ Similarly, Lebanon passed a landmark law in 2011 with strong stipulations to ban smoking in all public places. However, opposition from the hospitality sector and a weak enforcement contributed to stifling this ban. ${ }^{12}$ Egypt prohibited smoking, including waterpipe, in health and educational facilities in addition to governmental buildings, transportation and sporting club. However, the implementation is still a challenge. ${ }^{13}$

\section{ARTICLE 9. REGULATION OF THE CONTENTS OF TOBACCO PRODUCTS}

Although limited information is available on the regulation of the content of tobacco products in the region, several countries (eg, Jordan, Iraq and the GCC countries) have laws that regulate the additives and ingredients in tobacco products. ${ }^{14}$ In addition, legislation and regulation to address novel tobacco products is lacking in many EMR countries.

\section{ARTICLE 11. PACKAGING AND LABELLING OF TOBACCO PRODUCTS}

Health warnings on cigarettes packs are mandated in all EMR countries, except Somalia. The region ranks second after Southeast Asia, which has health warnings mandated in all its countries. Pictorial health warning labels have been implemented in several EMR countries with variations in their size relative to cigarette packs. For example, pictorial health warnings occupy $60 \%$ of the pack in Pakistan, but only 40\% of the pack in Jordan. ${ }^{14}$ In 2019 , Saudi Arabia became the first EMR country and among the first worldwide to introduce plain packaging on tobacco packs. ${ }^{15}$ In Lebanon, the textual and pictorial health warnings should occupy $40 \%$ of the overall area of any tobacco pack including waterpipe while in Egypt it should occupy $50 \%$ of the pack. ${ }^{13}$

\section{ARTICLE 12. EDUCATION, COMMUNICATION, TRAINING AND PUBLIC AWARENESS}

Tobacco control mass media campaigns have been conducted across most EMR countries. The number of EMR countries that have conducted at least one nationwide mass media campaign is high compared with other regions. For example, comprehensive tobacco control campaigns were launched in Iraq and Qatar between 2018 and 2019 in partnership with governmental and non-governmental organisations. ${ }^{16} 17$ Civil society has a track record of advocating for tobacco control in the region, including in Bahrain, Lebanon and the occupied Palestinian territory. ${ }^{3}$

\section{ARTICLE 13. TOBACCO ADVERTISING, PROMOTION AND SPONSORSHIP}

All countries in the region, except Somalia, have adopted partial or complete bans on tobacco advertising, promotion and sponsorship in collaboration with non-governmental bodies. Bans on advertising at the point of sale, and through the Internet, printed media and outdoor advertising were key areas overlooked in some countries resulting in partial bans. Overall compliance with bans on promotion and sponsorship is generally high. For example, Bahrain was the first among GCC countries to ban tobacco advertising, promotion and sponsorship in collaboration with non-governmental bodies in 2011, ${ }^{1}$ while Oman implemented a successful ban on all point-of-sale advertisements in $2017 .^{18} 19$

\section{ARTICLE 14. DEMAND REDUCTION MEASURES CONCERNING TOBACCO DEPENDENCE AND CESSATION}

Bahrain and Saudi Arabia were the first in the region to establish smoking cessation services at the national level in 2004 and 2011, respectively. Saudi Arabia implemented specialised cessation clinics, a national quitline and electric mobile applications to facilitate access to cessation services. ${ }^{20}$ Currently, smoking cessation medications are fully covered in Bahrain, Jordan, Kuwait, Qatar and Saudi Arabia. In Lebanon and Tunisia where smoking rates are among the highest in the region, smoking cessation programmes remain limited, ${ }^{21}$ and waterpipe smoking is insufficiently addressed in these measures despite WHO recommendations.

\section{ARTICLE 15. ILLICIT TRADE IN TOBACCO PRODUCTS}

Approximately half of the countries in the EMR are either signatories or parties to the Protocol to Eliminate Illicit Trade in Tobacco. As of September 2020, formal parties include Egypt, Iraq, Iran, Kuwait, Pakistan, Qatar and Saudi Arabia. Five other countries (Libya, Sudan, Syria, Tunisia and Yemen) are signatories to the Protocol. ${ }^{22}$ All formal parties in the region, except Egypt and Pakistan, completed their reporting obligations by 2020 .

\section{ARTICLE 20. RESEARCH, SURVEILLANCE AND EXCHANGE OF INFORMATION}

As of 2019, only five EMR countries have complete tobacco monitoring policies, with regularly collected representative data. ${ }^{23}$ According to the WHO, EMR countries have moderate to complete monitoring policies (such as Egypt, Iraq, Kuwait and Lebanon), and six countries have minimal monitoring policies (Bahrain, Jordan, Mauritania, Palestine, Saudi Arabia and Yemen) or no monitoring policies (Syria and Libya). ${ }^{1}$ Approximately half of the countries in the region have surveyed their adult or youth populations in the past 5 years.

Along with the tobacco epidemic facing many countries, there are significant knowledge gaps related to tobacco control research. Research from the region is predominately descriptive, with less focus on interventional studies. For example, despite the rise in waterpipe smoking prevalence, research on effective interventions to address this trend is limited. ${ }^{24}$ Besides the scarcity of research to address the evolving tobacco use landscape in the region, there is a need for stronger surveillance systems to monitor changes in tobacco use patterns and inform tobacco control priorities.

In addition, the population-level impact of implemented tobacco control measures is not consistently evaluated. Tobacco control is multifaceted; therefore, a multidisciplinary approach and sustained implementation are key to the continued success of national tobacco control programmes. Monitoring the implementation of FCTC programmes and policies requires reliable surveillance systems with timely, high-quality data using standardised methodology. Measuring compliance of tobacco surveillance to standard methods of data collection and reporting on indicators is a major gap in the region. Parties responsible for the implementation of the FCTC require support to continue to prioritise tobacco control efforts in the midst of competing agendas. Establishing surveillance systems requires multinational collaborations that share strategies, 
successes, challenges and resources. Such efforts could help in identifying context-specific factors that facilitate policy implementation. The continuous provision of evidencebased knowledge will only bridge the gap between policy and practice if it includes sufficient attention to and capacity for appropriate implementation of the most effective policies and programmes for each setting.

Despite the progress in tobacco control legislation, there have been setbacks in enforcement. ${ }^{7}$ Enacting legislation does not necessarily lead to policy implementation. The common thread is lack of capacity within responsible parties for sustained implementation, and insufficient political support to negotiate collaboration and support from other stakeholders and to elicit compliance from the hospitality sector. ${ }^{12}$ As a result, EMR countries continue to fall short on protecting their populations from the harm of tobacco use..$^{25}$

With strong global support for implementing the FCTC and significant gaps in implementation capacity and enforcement across the region, there has never been a greater need to expand the application of dissemination and implementation science, particularly in a region where the burden of tobacco use is excessive. Dissemination and implementation science provides a crucial set of tools for informing and supporting tobacco control across and extending the reach and impact of the FCTC with a goal of ultimately reducing the burden of tobacco-related disease and death in the region. ${ }^{26}$

\section{Twitter Rima T Nakkash @rimanakkash and Randah R Hamadeh @UmSamar}

Contributors All authors were involved in the conceptualisation of the paper. NMEA-R drafted the manuscript, YSK and HAR prepared the prevalence part. AM, RTN and RGS prepared the policy part. RRH and HBR revised the manuscript. All coauthors have read the final version of the paper.

Funding This work was funded in part by the International Development Research Centre in partnership with Cancer Research UK (Grant number 108821).

Disclaimer The funding agency played no role in study design, in the collection, analysis and interpretation of data, in the writing of the report and in the decision to submit the article for publication. The content is solely the responsibility of the authors and does not necessarily represent the official views of the funding agency.

\section{Competing interests None declared.}

\section{Patient consent for publication Not applicable.}

Ethics approval This study does not involve human participants.

Provenance and peer review Commissioned; externally peer reviewed.

Supplemental material This content has been supplied by the author(s). It has not been vetted by BMJ Publishing Group Limited (BMJ) and may not have been peer-reviewed. Any opinions or recommendations discussed are solely those of the author(s) and are not endorsed by BMJ. BMJ disclaims all liability and responsibility arising from any reliance placed on the content. Where the content includes any translated material, BMJ does not warrant the accuracy and reliability of the translations (including but not limited to local regulations, clinical guidelines, terminology, drug names and drug dosages), and is not responsible for any error and/or omissions arising from translation and adaptation or otherwise.

\section{ORCID iDs}

Niveen M E Abu-Rmeileh http://orcid.org/0000-0003-0831-9692

Aya Mostafa http://orcid.org/0000-0002-2803-2608

Rima T Nakkash http://orcid.org/0000-0001-8800-5591

Randah R Hamadeh http://orcid.org/0000-0002-4205-1354

Ramzi George Salloum http://orcid.org/0000-0002-8139-2418

\section{REFERENCES}

1 World Health Organization. WHO report on the global tobacco epidemic 2021: addressing new and emerging products, 2021.

2 Organization WH. Tobacco industry interference with tobacco control, 2009.

3 Maziak W, Nakkash R, Bahelah R, et al. Tobacco in the Arab world: old and new epidemics amidst policy paralysis. Health Policy Plan 2014;29:784-94.

4 Raz D. Youth in the Middle East and North Africa. Arab Barometer 2019.

5 Hamadeh RR, Lee J, Abu-Rmeileh NME, et al. Gender differences in waterpipe tobacco smoking among university students in four eastern Mediterranean countries. Tob Induc Dis 2020:18:1-12.

6 AkI EA, Ward KD, Bteddini D, et al. The allure of the waterpipe: a narrative review of factors affecting the epidemic rise in waterpipe smoking among young persons globally. Tob Control 2015;24 Suppl 1:113-21.

7 Chung-Hall J, Craig L, Gravely S, et al. Impact of the who FCTC over the first decade: a global evidence review prepared for the impact assessment expert group. Tob Control 2019:28:s119-28.

8 Tobacco Tactics. University of Bath. Eastern Mediterranean region. Available: https:// tobaccotactics.org/wiki/eastern-mediterranean-region/ [Accessed 27 Mar 2021].

9 Chaloupka F, Drope J, Siu E. Tobacconomics Cigarette Tax Scorecard. Chicago, IL, USA: Institute for Health Research and Policy, University of Illinois Chicago, 2020.

10 Chalak A, Nakkash R, Abu-Rmeileh NME, et al. Own-price and cross-price elasticities of demand for cigarettes and waterpipe tobacco in three Eastern Mediterranean countries: a volumetric choice experiment. Tob Contro/ 2021. doi:10.1136/ tobaccocontrol-2021-056616. [Epub ahead of print: 30 Jun 2021].

11 Burki TK. Tobacco control in Jordan. Lancet Respir Med 2019;7:386.

12 Nakkash RT, Torossian L, El Hajj T, et al. The passage of tobacco control law 174 in Lebanon: reflections on the problem, policies and politics. Health Policy Plan 2018:33:633-44.

13 Organization WH. An overview of global regulatory practices in controlling waterpipe tobacco use, 2019

14 Muggli ME, Zheng A, Liberman J, et al. Tracking the relevance of the WHO Framework Convention on Tobacco Control in legislation and litigation through the online resource, Tobacco Control Laws. Tob Control 2014;23:457-60.

15 World Health Organization. Regional office for the eastern Mediterranean. Tobacco free initiative. Newsroom. Saudi Arabia adopts plain packaging on tobacco products: a groundbreaking step for tobacco control | news | TFI. Available: http://www.emro. who.int/tfi/news/saudi-arabia-adopts-plains-packaging-on-tobacco-products-agroundbreaking-step-for-tobacco-control.html [Accessed 27 Mar 2021].

16 Hamad Medical Coporation. HMC-Led Anti-Smoking Campaign Continues to Focus on Schools and Local Businesses. Available: https://www.hamad.qa/EN/news/2018/may/ Pages/HMC-Led-Anti-Smoking-Campaign-Continues-to-Focus-on-Schools-and-LocalBusinesses.aspx [Accessed 27 Mar 2021].

17 The National comprehensive anti-smoking campaign. Ministry of Health. Iraq, 2019 Available: https://www.facebook.com/Diwaniya.Health/posts/1786519394811879 [Accessed 27 Mar 2021].

18 WHO Framework Convention on Tobacco Control. Oman: points of sale advertising banned. Available: https://untobaccocontrol.org/impldb/oman-points-of-saleadvertising-banned/ [Accessed 27 Mar 2021].

19 Al-Lawati J, Mabry RM, Al-Busaidi ZQ. Tobacco control in Oman: it's time to get serious! Oman Med J 2017:32:3-14.

20 Ministry of Health. Kingdom of Saudi Arabia. MOH Portal. The Ministry. MOH Initiatives \& Projects. Anti-Smoking Clinics, 2021.

21 Romani M, Nakkash R, Jawhar S, et al. Implementation of a free smoking-cessation program in a Lebanese academic medical center. Tob Induc Dis 2020;18:1-5.

22 United Nations Treaty Collection. Depository. Status of Treaties. Chapter IX. Health. 4. A protocol to eliminate illicit trade in tobacco products. Available: https://treaties.un. org/Pages/ViewDetails.aspx?src=TREATY\&mtdsg_no=IX-4-a\&chapter=9\&clang=_en [Accessed 27 Mar 2021].

23 World Health Organization. WHO report on the global tobacco epidemic, 2019: offer help to quit tobacco use. World Health Organization, 2019

24 Hamadeh RR, Borgan SM, Khabsa J, et al. Tobacco research in the eastern Mediterranean region: a scoping review of published studies from seven countries. $J$ Community Health 2021;46:225-31.

25 Bader RK, Shihab RA, Al-Rimawi DH, et al. Informing tobacco control policy in Jordan: assessing the effectiveness of pictorial warning labels on cigarette packs. BMC Public Health 2017;18:84

26 Salloum RG, Parascandola M, LeLaurin JH, et al. The role of dissemination and implementation research in global tobacco control: a report from the American Society of Preventive Oncology. Cancer Epidemiol Biomarkers Prev 2020;29:2740-3. 